\title{
Energy Trading in the Smart Grid: From End-user's Perspective
}

\author{
Shengbo Chen*, Ness B. Shroff ${ }^{\dagger}$ and Prasun Sinha ${ }^{\ddagger}$ \\ ${ }^{*}$ Department of ECE, The Ohio State University \\ $\dagger \ddagger$ Department of CSE, The Ohio State University \\ Email: $\left\{{ }^{*}\right.$ chens, ${ }^{\dagger}$ shroff $\} @$ ece.osu.edu, ${ }^{\dagger}$ prasun@ @cse.ohio-state.edu
}

\begin{abstract}
The smart grid is expected to be the next generation of electricity grid. It will enable numerous revolutionary features, that allow end-users, such as homes, communities, or businesses, to harvest renewable energy and store the energy in a local battery, which could act as a microgeneration unit. In addition, the customers could be provided with dynamic electricity pricing options under which the electricity price adjusts over a relatively small time scale, e.g., every hour, in response to the changing energy usage of the grid. Consequently, similar to the stock market, it presents an opportunity for an end-user to make profit by trading energy with the grid. More specifically, in this paper, we investigate the profit maximization problem for an end-user that is equipped with renewable energy harvesting devices and a battery, such that the user can buy/sell the energy from/to the grid by leveraging the varying price and the battery storage ability. The resulting algorithm performs arbitrarily close to the optimum without requiring future information of energy demands, electricity prices and the renewable energy arrival process. We validate our results through trace driven simulations. Based on observations from the simulations, we discuss the potential applicability of our scheme in both a typical home and a community setting by taking into account the wear-and-tear cost of the battery.
\end{abstract}

\section{INTRODUCTION}

The smart grid, the next-generation electricity grid, endows both suppliers and consumers with full visibility and pervasive control over their assets and services [1]. The smart grid features numerous revolutionary concepts compared to its predecessor. For instance, renewable energy sources (e.g., solar or wind) [2] can be incorporated into the grid. The grid can be equipped with energy harvesting and storage devices (battery), which is also referred to as "microgeneration unit". In addition, in the context of the smart grid, end-users may be given an option under which the electricity price adjusts over a relatively small time scale, e.g., every hour, in response to the varying energy usage of the grid. [3]. Current control policies are unable to fully exploit these new features. Thus, more delicate controls are urgently required in order to make efficient use of these new features in the smart grid.

In this paper, we focus on an end-user in the smart grid, such as a home, a community, or a business that is equipped with renewable energy devices. The renewable energy devices consist of an energy storage battery and an energy harvesting device. Renewable energy can be harvested and stored in the battery. The energy demand at the user site is a stochastic process that is met by either drawing energy from the grid or the battery, as shown in Fig. 1. The battery is also allowed

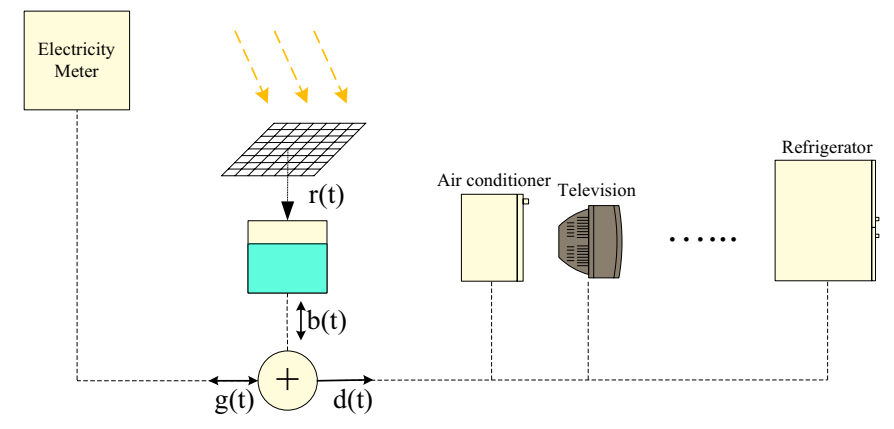

Fig. 1. Energy Demand and Supply (please refer to Table I for notations)

to recharge by drawing energy from the grid, which can be purchased and stored, based on fluctuating electricity prices.

In this work, we are interested in developing an optimal energy trading algorithm that maximizes the total profit while satisfying the user's instantaneous energy demand. To put it simply, similar to the stock market, the varying electricity price and energy storage capability present an opportunity for an end-user to make profit via buying/selling the energy from/to the grid.

\section{A. State-of-the-art}

In the area of the smart grid, there have been prior works that focus on energy scheduling problems. In our previous work [4], we have considered time-varying electricity prices and renewable energy devices to develop an electricity appliance scheduling policy in order to minimize the total cost under both average and hard delay constraints. In [5], the authors investigate the problem of optimal energy demand scheduling subject to deadlines, in order to minimize the cost. They consider both preemptive and non-preemptive tasks. They also consider online dynamic scheduling schemes, where two heuristic algorithms are proposed. However, the algorithms are shown to achieve asymptotic optimality only as the delay constraints tend to be arbitrarily loose. In [6], the authors develop an energy allocation algorithm to minimize the total electricity cost. However, they do not allow renewable energy to be stored for future usage. Other works have adopted dynamic programming techniques, e.g. [7]. In these works, optimality can be achieved only if the distribution of the energy demand is known a priori. There have also been 


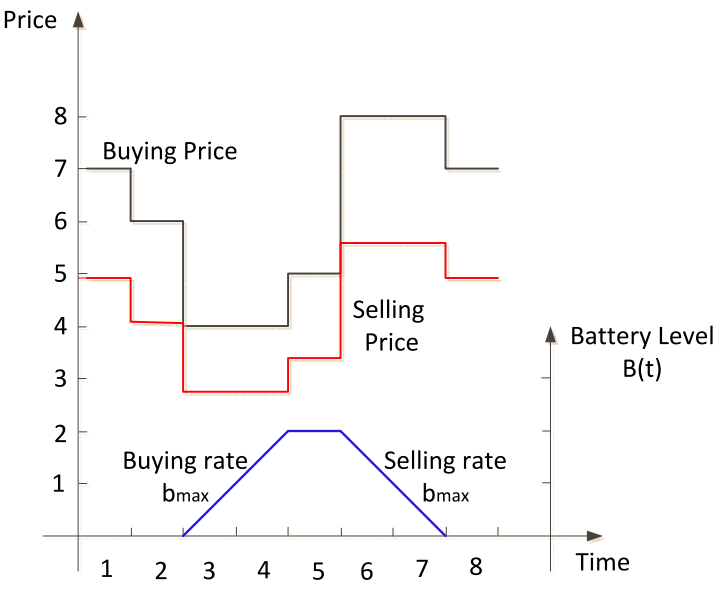

Fig. 2. An example of energy trading

other works that have formulated problems using game theory, e.g., [8]-[10]. The authors in [11] consider an energy trading problem using game theory in the Vehicle-to-Grid problem. In the context of stock trading policies, the authors in [12] develop a dynamic trading algorithm to maximize the total profit. However, unlike the battery constraint considered in this paper, there are no hard constraints on the battery sizes in their formulations.

\section{B. Our Contributions}

In this paper, we address the energy trading problem from an end-user's perspective. The end-user is assumed to have a battery that can be recharged with renewable energy. To the best of our knowledge, this is the first work that explores energy trading opportunities for end-users in the area of the smart grid. Having a battery is crucial for trading the energy since, generally speaking, the battery can draw energy from the external grid when the electricity price is low and can discharge energy when the price is high. Fig. 2 shows a simple example. The electricity price is depicted by the black curve and the selling price is depicted by the red curve. We can see that the buying prices in time-slots 3 and 4 are lower than the selling prices in time-slots 6 and 7. Therefore, the optimal strategy is to buy energy at the highest possible rate during slots 3 and 4, and to sell the stored energy during slots 6 and 7 , on condition that there is sufficient space in the battery to store the energy. The battery level dynamics are shown by the blue curve. Furthermore, renewable energy also brings about new challenges. First, renewable energy is highly timevarying, making it challenging to incorporate. Second, it adds more stochastic fluctuation to the energy trading problem.

We summarize our main contributions as follows:

1). We consider the energy trading problem in the smart grid by leveraging the time-varying electricity prices and an energy storage battery. To the best of our knowledge, this is the first work that considers this problem.

2 ). We propose a simple algorithm that achieves performance that can be made arbitrarily close to the optimum. Note that our algorithm does not require future knowledge of the

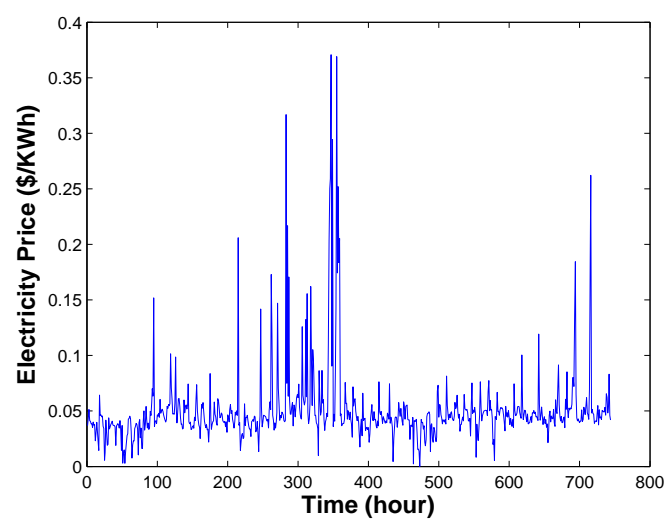

Fig. 3. One-hour average spot market price during the month of 1/1/20071/31/2007 for Los Angeles Area (Zone LA1) from CAISO [3]

energy demands, electricity prices, and the renewable energy arrival process.

3). We validate our algorithm using real traces to compute the realistic profits. We show that our algorithm indeed achieves good performance under various system parameter settings. Based on the observations from the simulations, we discuss the potential applicability of our scheme for both a typical home and a community setting by taking into account the wear-and-tear cost of the battery.

Our paper is organized as follows: In Section II, we discuss our system model. In Section III, we formulate the profit maximization problem. In Section IV, we develop the energy trading algorithm and show its performance. After presenting simulation results in Section $\mathrm{V}$, we discuss the potential applicability of our scheme in Section VI. We conclude our paper in Section VII.

\section{SYSTEM MODEL}

We consider an end-user that is connected to the external smart grid. Time is assumed to be slotted. Let $P(t)$ denote the price of electricity in time slot $t$. As an example, Fig. 3 shows the average one-hour spot market prices for the Los Angeles area obtained from CAISO [3]. Let $d(t)$ represent the energy demand of the end-user in time slot $t$. Let $r(t)$ denote the harvested renewable energy that is stored in the battery at time slot $t$. Note that $r(t)$ is the actual harvested renewable energy that has already excluded the energy loss occurring during the energy harvesting process. For simplicity of exposition, we assume that $r(t)$ amount of energy gets stored in the battery at the end of the slot $t$. Initially, we assume that the battery has infinite capacity. We later show that our algorithm only requires a reasonable sized finite battery.

Note that part of the energy demand $d(t)$ can be met by drawing energy from the battery, while the remaining is drawn from the grid. Let $g(t)$ and $b(t)$ represent the amount of energy that is drawn from the external grid and the battery in time slot $t$, respectively. Because the supply always needs to balance the 
demand, as shown in Fig. 1, we have

$$
d(t)=g(t)+b(t)
$$

In addition, we also allow the battery to charge energy from the grid, which means that $b(t)$ could be negative. In particular, the battery discharges/charges energy if we have $b(t) \gtrless 0$. We let $b_{\max }$ denote the maximum allowed amount of energy for charging the battery from the grid or discharging from the battery in one time slot, which reflects the physical constraints on the wires. We use $B(t)$ to denote the battery level at the beginning of time slot $t$, and the energy dynamics can be formulated as follows:

$$
B(t+1)=B(t)-b(t)+r(t) .
$$

The constraints on $b(t)$ are given by

$$
\begin{aligned}
& |b(t)| \leq b_{\max }, \\
& b(t) \leq B(t),
\end{aligned}
$$

where the second constraint means that the allocated energy from the battery should be less than or equal to the current available energy in the battery. We assume that the electricity price, the amount of harvested renewable energy and energy demand, $(P(t), r(t), d(t))$, are all i.i.d and non-negative over time slots. We denote $\bar{r} \triangleq \mathbb{E}[r(t)]$ and $\bar{d} \triangleq \mathbb{E}[d(t)]$. It is worth pointing out that our results can be generalized to the case when they are Markovian through a similar argument as in [13], which we omit here due to space limitation.

\section{A. Energy Selling Model}

We can see that energy is sold to the grid if $g(t)<0$. In this paper, we assume that the selling price is $\beta P(t)$, where $\beta$ is a constant between 0 and 1 . The reason why the parameter $\beta$ is less than one is that there exists energy loss when the utility company tries to harness the energy from the enduser's battery, such as the AC/DC conversion loss and the transmission line loss. Further, given that the utility company has its own costs, it would buy energy at a lower rate than what it sells for at any given time. This model assumes that the selling price is always less than the buying price in each time slot. It also implies that buying and selling energy must not occur simultaneously, i.e., in the same time slot, because it would lead to an obvious loss for the customer due to the price gap. Therefore, when energy is being drawn from the grid, the cost is given by

$$
P(t)(g(t))^{+}=P(t)(d(t)-b(t))^{+},
$$

where $(a)^{+} \triangleq \max \{a, 0\}$. In contrast, when selling energy to the grid, the profit is given by

$$
\beta P(t)(g(t))^{-}=\beta P(t)(d(t)-b(t))^{-},
$$

where $(a)^{-} \triangleq \max \{-a, 0\}$.

The key notations are summarized in Table I.
TABLE I

NOTATIONS

\begin{tabular}{|l||l|}
\hline$P(t)$ & Electricity price in time slot $t$ \\
$d(t)$ & Energy demand in time slot $t$ \\
$g(t)$ & Energy drawn from the grid in time slot $t$ \\
$b(t)$ & Energy drawn from the battery in time slot $t$ \\
$r(t)$ & Harvested renewable energy in time slot $t$ \\
$B(t)$ & Battery level in time slot $t$ \\
\hline
\end{tabular}

\section{PROBLEM ForMULATION}

Our goal in this paper is to maximize the long-term average profit. We thus formulate the profit maximization problem as follows:

$$
\begin{aligned}
& \text { Problem A: } \max _{b(t)} \lim _{T \rightarrow \infty} \frac{1}{T} \sum_{t=1}^{T} \mathbb{E}\left[\beta P(t)(d(t)-b(t))^{-}\right. \\
& \left.-P(t)(d(t)-b(t))^{+}\right] \text {, } \\
& \text { s.t. (3), (4), }
\end{aligned}
$$

where the expectation is with respect to the distribution of $(P(t), r(t), d(t))$.

Now we aim at finding an optimal solution to Problem A. We use the Lyapunov optimization approach [13] to solve it. We let $c^{o p t}$ denote the maximum profit achieved by the solution to Problem $\mathbf{A}$ and $\tilde{c}$ denote the maximum profit achieved by the solution to Problem A without the constraint Eqn. (4). Clearly, $\tilde{c}$ forms an upper bound on $c^{o p t}$, i.e., $\tilde{c} \geq c^{o p t}$.

Next, we will first show that $\tilde{c}$ is achievable under some stationary and randomized policy (defined later). We then show that our proposed scheme achieves a profit greater than $\tilde{c}-\epsilon$, and thus greater than $c^{o p t}-\epsilon$, where $\epsilon$ could be an arbitrary positive parameter.

\section{A. Optimal Stationary and Randomized Policy}

In this subsection, we first consider a class of stationary and randomized policies. That is, the control action $b(t)$ in every time slot depends purely on the current state $(P(t), r(t), d(t))$ and is independent of the battery level $B(t)$. Observe that this control scheme may not always satisfy Eqn. (4), which is required by any practical policy. Furthermore, these stationary and randomized policies are allowed to have future knowledge, such as the distribution of the electricity prices, the energy demands and the renewable energy process. Through a similar argument as in [12], we can also show that there exists a stationary and randomized policy for which a profit of $\tilde{c}$ is achievable using the following lemma.

Lemma 3.1: A profit of $\tilde{c}$ can be achieved by a stationary and randomized policy, that is, the control action $\tilde{b}(t)$ at each time slot is only a function of $(P(t), r(t), d(t))$. In particular, 
we have

$$
\begin{aligned}
& \tilde{c}=\lim _{T \rightarrow \infty} \frac{1}{T} \sum_{t=1}^{T} \mathbb{E}\left[\beta P(t)(d(t)-\tilde{b}(t))^{-}\right. \\
&\left.-P(t)(d(t)-\tilde{b}(t))^{+}\right], \\
& \mathbb{E}[\tilde{b}(t)]=\bar{r}
\end{aligned}
$$

where $\bar{r}$ is the average energy replenishment rate.

Proof: The proof argument follows the argument in [12], We refer to our technical report [14] for the details.

In the lemma, Eqn. (8) implies that the average allocated energy from the battery is equal to the average harvested energy.

\section{Energy Trading Algorithm}

Notice that the optimal stationary and randomized policy requires future information and is therefore impractical. So, in this section, we propose a dynamic policy and show that its performance can be arbitrarily close to the optimum over an infinite time horizon, i.e., $T \rightarrow \infty$.

\section{A. Dynamic Energy Trading Algorithm}

Our goal is to develop a dynamic energy trading algorithm, which can achieve optimality without requiring future knowledge. Before we proceed, we define the parameters that will be used in our scheme. Define a constant $P_{\max }$ as the highest electricity price over all time slots, i.e., $P_{\max }=\max _{t} P(t)$, and also define a constant parameter $\theta=V P_{\max }+b_{\max }$, where $V$ is a positive parameter to be chosen as desired to affect the proximity to the optimal time average profit. We first present our algorithm and then prove its performance.

\section{ETA (Energy Trading Algorithm):}

We define $\alpha(t)=V P(t)+B(t)-\theta$ and $\gamma(t)=V \beta P(t)+$ $B(t)-\theta$. Observe that $\alpha(t)>\gamma(t)$ always holds by the definitions. Thus, there are only three possibilities: both are non-negative, both are negative, $\alpha(t)$ is non-negative and $\gamma(t)$ is negative.

In each time slot $t$, based on the electricity price $P(t)$, the battery level $B(t)$ and the energy demand $d(t)$, the battery charging/discharging energy is given by:

- If $\alpha(t)>\gamma(t) \geq 0$, we set

$$
b^{*}(t)=b_{\max } .
$$

- If $0>\alpha(t)>\gamma(t)$, we set

$$
b^{*}(t)=-b_{\max } .
$$

- If $\alpha(t) \geq 0>\gamma(t)$, we set

$$
b^{*}(t)=\min \left\{b_{\max }, d(t)\right\} .
$$

Note that Eqns. (9), (10) and (11) correspond to the three cases, respectively. We also notice that ETA is very simple and therefore has a very low computational overhead. Before we proceed to the performance analysis, we would like to discuss the ETA scheme and its intuition.
$1)$. We can see that when $B(t)<b_{\max }$, it always has $V P(t)+B(t)-\theta<0$ by the definition of $\theta$. Hence, from Eqn. (10), the battery always draws energy from the grid, namely $b(t)=-b_{\max }$, when the battery level is less than $b_{\max }$. This implies that our scheme ETA guarantees that when the battery discharges, i.e., $b(t)>0$, there is always enough energy in the battery, i.e., $B(t) \geq b_{\max }$. Therefore, the energy constraint $b(t) \leq B(t)$, i.e., Eqn. (4), is never violated.

2). From Eqn. (9), we can see that when $V \beta P(t)+B(t)-$ $\theta \geq 0$, we should always set $b^{*}(t)=b_{\max }$, which is the maximum allowed rate of discharging from the battery. When $d(t) \geq b_{\max }$, the decision $b^{*}(t)=b_{\max }$ means that $b_{\max }$ out of the energy demand $d(t)$ is from the battery. When $d(t)<$ $b_{\max }$, the decision is to sell the extra amount $b_{\max }-d(t)$ of energy to the grid. The intuition is that when either the electricity price $P(t)$ or the battery level $B(t)$ is high, we can make profit or reduce cost by drawing energy from the battery at the most aggressive speed.

3). Consider the case of Eqn. (10), we can see that both the electricity price $P(t)$ and the battery level $B(t)$ are low. The decision is $b^{*}(t)=-b_{\max }$, i.e., buying energy from the grid. The intuition is that we should buy energy from the external grid when the electricity price is low and the battery level is low.

4). Comparing Eqn. (11) with Eqn. (9) and Eqn. (10), we can see that the electricity price $P(t)$ and the battery level $B(t)$ are in between the previous two cases, i.e., not as high when compared to the case of Eqn. (9) and not as low when compared to the case of Eqn. (10). Its corresponding decision $b^{*}(t)=\min \left\{b_{\max }, d(t)\right\}$ is also in-between, which implies that we should just draw energy from the battery only to meet the energy demand without selling or buying energy.

\section{B. Performance Analysis}

We define a Lyapunov function $L(t)=\frac{1}{2}(B(t)-\theta)^{2}$. The intuition behind it is that, by minimizing the drift of the Lyapunov function, we force $B(t)$ to approach $\theta$. We also define a constant $r_{\max } \triangleq \max _{t} r(t)$, which is the maximum stored energy in one time slot. We assume two homogeneous wires, which is the one connecting the battery and the grid, as well as the one connecting the battery and the solar panel. Note that $r_{\max } \leq b_{\max }$. The reason is simply because in any time slot, the maximum amount of stored energy, $r_{\max }$, should be no greater than the maximum amount of allowed energy through the wire, $b_{\max }$.

The conditional Lyapunov drift is given by $\mathbb{E}\{(L(t+1)-$ $L(t) \mid B(t)\}$. We will first show some properties of the drift via the following lemma.

Lemma 4.1: The conditional Lyapunov drift satisfies

$$
\begin{aligned}
& \mathbb{E}\{(L(t+1)-L(t) \mid B(t)\}- \\
& V \mathbb{E}\left[\beta P(t)(d(t)-b(t))^{-}-P(t)(d(t)-b(t))^{+} \mid B(t)\right] \\
& \leq D+(V P(t)+B(t)-\theta) \mathbb{E}\left[(d(t)-b(t))^{+} \mid B(t)\right] \\
& -(V \beta P(t)+B(t)-\theta) \mathbb{E}\left[(d(t)-b(t))^{-} \mid B(t)\right] \\
& -(B(t)-\theta)(\bar{d}-\bar{r}),
\end{aligned}
$$


where $D \triangleq \frac{1}{2} r_{\text {max }}^{2}+\frac{1}{2} b_{\text {max }}^{2}$.

Proof: We refer to our technical report [14] for the details.

Next, we have the following lemma:

Lemma 4.2: Our energy trading policy ETA, i.e., Eqns. (9),(10),(11), minimizes the RHS of Eqn. (12).

Proof: We refer to our technical report [14] for the details.

Now we are ready to prove that our scheme ETA can be made to perform arbitrarily close to the optimum via the following theorem.

Theorem 4.3: By setting $B(0)=\theta$, our scheme ETA has the following properties:

1) The battery level $B(t)$ is always bounded, which satisfies:

$$
B(t) \leq \theta+b_{\max }+r_{\max }, \forall t .
$$

2) The time average profit achieved by ETA satisfies:

$$
\begin{aligned}
& \limsup _{T \rightarrow \infty} \frac{1}{T} \sum_{t=1}^{T} \mathbb{E}\left[\beta P(t)\left(d(t)-b^{*}(t)\right)^{-}\right. \\
& \left.-P(t)\left(d(t)-b^{*}(t)\right)^{+}\right] \\
& \geq c^{o p t}-\frac{D}{V} .
\end{aligned}
$$

Proof: We refer to Appendix A for the proof.

Part 1 in Theorem 4.3 shows that our scheme only requires a finite battery size, and part 2 shows that the profit achieved by our algorithm can be made to be arbitrarily close to $c^{o p t}$, i.e., maximum profit achieved by the solution to Problem $\mathbf{A}$, as the parameter $V$ becomes sufficiently large. It is worth pointing out that the algorithm does not require future knowledge of the energy demands, electricity prices or the renewable energy arrival process.

\section{Discussion}

1). If $\beta=1$, from the description of ETA, the decision $b(t)$ becomes $b(t)=b_{\max }$ if $V P(t)+B(t)-\theta \geq 0$ and $b(t)=-b_{\max }$ if $V P(t)+B(t)-\theta<0$, which means that $b(t)$ is independent of the energy demand $d(t)$. And our scheme will become a Bang-Bang control, i.e., $b(t)$ equals either $b_{\max }$ or $-b_{\max }$ in each time slot.

2). It is worth pointing out that the optimality of ETA also holds even if the energy replenishment rate is zero, that is, there is no energy harvesting device being used.

3). Since our focus here is on an end-user, like a home or a community, it is assumed here that the trading actions will not influence the electricity price. However, if the trading policy is adopted at scale by a large number of end-users (families or communities), it could lead to a significant impact on the electricity price. However, this issue is beyond the scope of this paper, and we plan to investigate it in our future work.
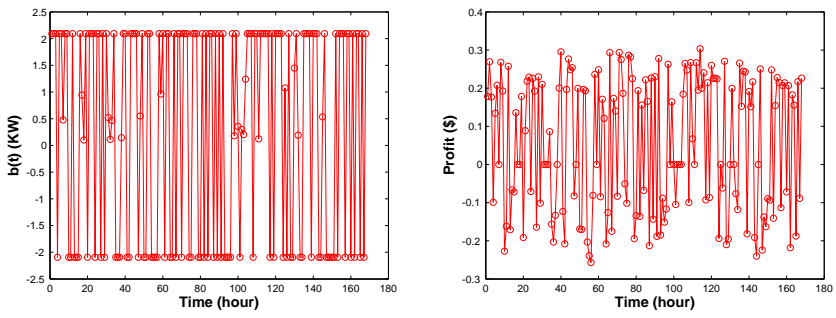

Fig. 4. (Left) Energy drawn from the battery in each one hour time slot. (Right) Profit in each one hour time slot.

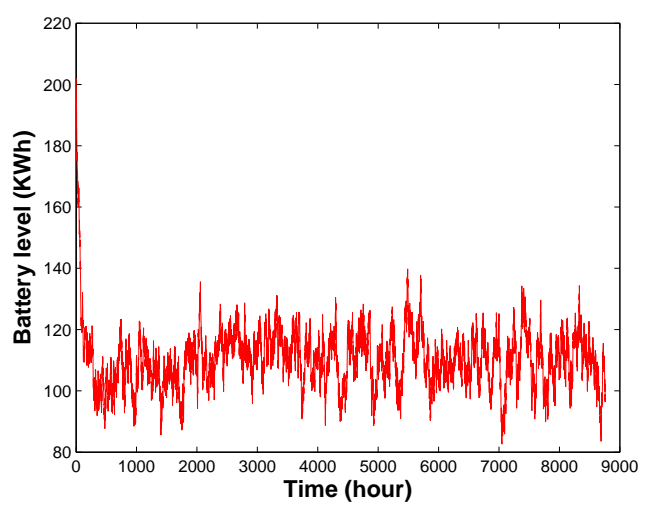

Fig. 5. Battery level in each one hour time slot

\section{CASE Study}

In this section, we evaluate the algorithm presented in the previous section using both synthetic and real traces. We first verify our theoretical results using synthetic data that corresponds to the i.i.d. model for $(P(t), r(t), d(t))$ and will later show that our scheme ETA also performs well under real traces. In both cases, each time slot is set to be one hour. The total period simulated is one year.

\section{A. Model Based}

First, in order to gain insights into the behavior of the algorithm, we use an i.i.d. model for $(P(t), r(t), d(t))$. The electricity price $P(t)$ is assumed to be uniformly distributed between $0.02 \$ / K W h$ and $0.15 \$ / K W h$. First, we use a simple exponential distribution to model the energy replenishment rate. The mean value is set to be $0.2 \mathrm{KW}$ per hour. The energy demand per hour is assumed to be a combination of a base $0.1 \mathrm{KW}$ and an exponentially distributed random variable with mean value of $0.3 \mathrm{KW} . b_{\max }$ is set to be $0.8 \mathrm{KWh}$. The parameters $\beta$ and $V$ are set to be 0.8 and 1000 , respectively.

We start by plotting the energy trading behavior and the profit accordingly. Fig. 4 shows the energy drawn from the battery, i.e., $b(t)$, versus time in a one-week period (on the left). It can be seen that $b(t)$ fluctuates significantly. Fig. 4 also shows the achieved profit in the same period (on the right), which corresponds to $b(t)$. In Fig. 5, the curve depicts the energy level in the battery, which is bounded. This result conforms to our analytical result in the previous section. 


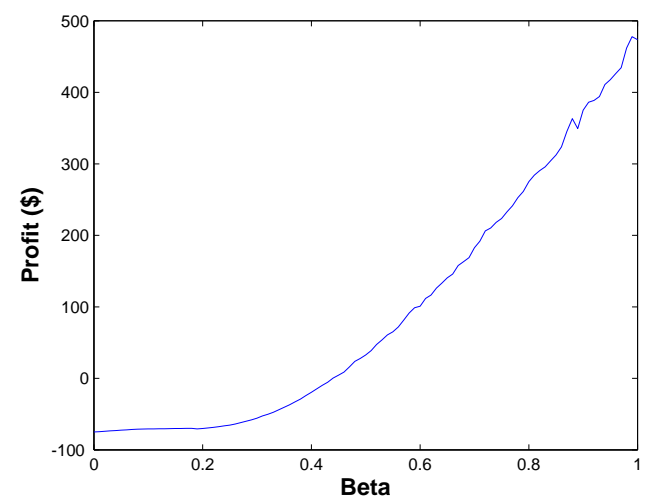

Fig. 6. Annual profit versus $\beta(\mathrm{V}=1000)$

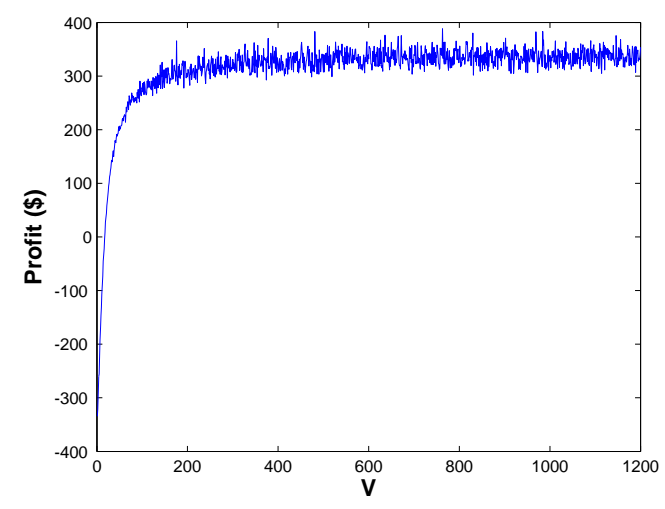

Fig. 7. Annual profit versus $V(\beta=0.8)$
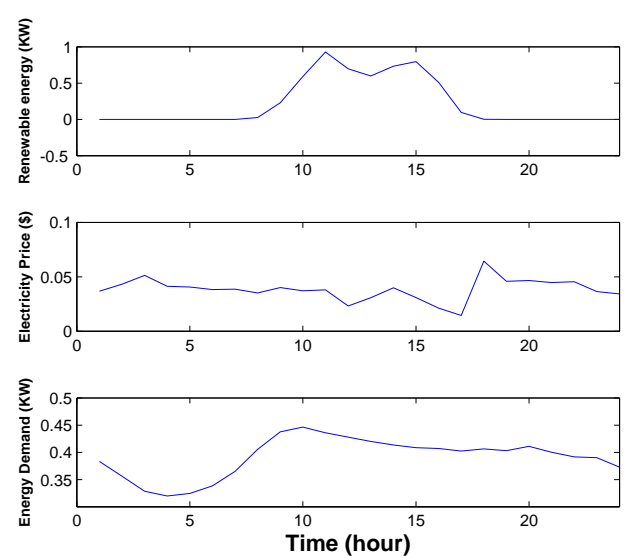

Fig. 8. Traces for solar energy, electricity prices and energy demands in one day $(1 / 1 / 2007)$

Next, we will show how the parameters $\beta$ and $V$ influence the profit. In Fig. 6, we show the profit achieved under varying $\beta$ while fixing $V=1000$. $\beta$ varies from 0 to 1 with step-size 0.01 . We can see that the total profit increases as $\beta$ becomes larger, which coincides with the intuition that the achieved profit increases if the selling price becomes higher. When $\beta$ is small, we can see that the total profit is even negative, which

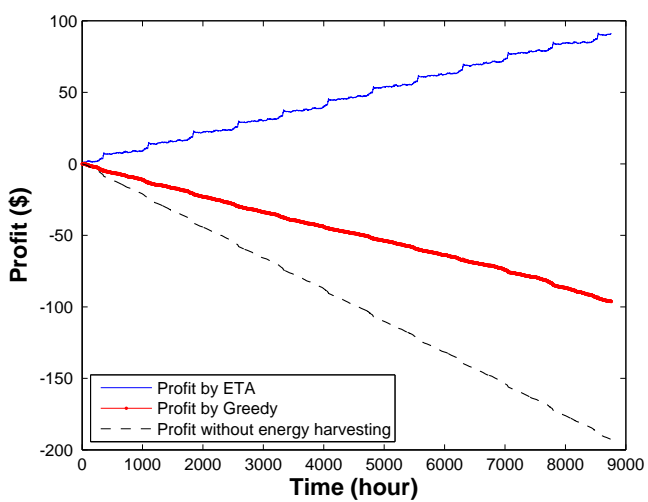

Fig. 9. Profit Comparison between ETA and Greedy

means that the money earned by selling energy cannot pay off the money spent on buying energy. In Fig. 7, we illustrate the relationship between the annul profit and the parameter $V$ while fixing $\beta=0.8$. It can be seen that when $V$ is small, the performance is poor and the profit becomes positive as $V$ grows beyond some threshold. The reason is that the term $\frac{D}{V}$ in Theorem 4.3 cannot be neglected when $V$ is small. We can also see that the curves converges as $V$ becomes larger, which is because the term $\frac{D}{V}$ in Theorem 4.3 becomes so small that the performance is close to the optimum.

\section{B. Trace Driven}

In the following simulations, we will show the performance of our scheme under real traces for electricity prices, energy demands and renewable energy process. For the electricity prices, we consider a one-year data set of average hourly spot market prices for Los Angeles Area (Zone LA1) from CAISO [3]. The period is $1 / 1 / 2007-12 / 31 / 2007$. We simulate the schemes using solar energy. We adopt raw data collected at the National Renewable Energy Laboratory [15] for the same period. One data point is collected per hour. The solar energy data set (Global 40-South LI-200) measures solar resource for collectors tilted 40 degrees from the horizontal and optimized for year-round performance. From the data, we can obtain the replenishment profile for solar energy, assuming that the enduser is equipped with a solar panel of an effective dimension $2000 \mathrm{~mm} \times 500 \mathrm{~mm}$. Regarding the traces of energy demands, we adopt raw data for one month [16] and repeat it for twelve times. The profile depicted in Fig. 8 shows the traces for solar energy profile, electricity prices, as well as energy demands in one day $(1 / 1 / 2007)$.

We compare our scheme with a heuristic scheme which works as follows. In each time slot, if the harvested energy is greater than the energy demand, the extra energy is sold. In contrast, if the harvested energy is less than the energy demand, the user buys energy from the external grid to bridge the gap. We call this scheme "Greedy". We simulate both schemes by setting $\beta=0.8$ and $V=1000$. We also show the cost curve if there is no energy harvesting devices. From Fig. 9, first, we can see that the profit achieved by ETA always 


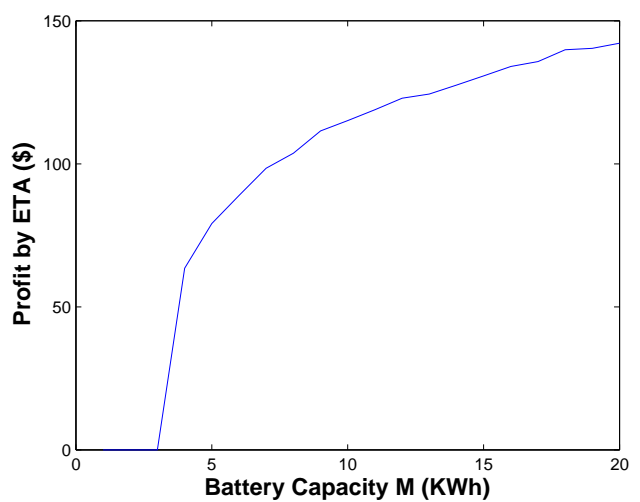

Fig. 10. Profit achieved by ETA in a home

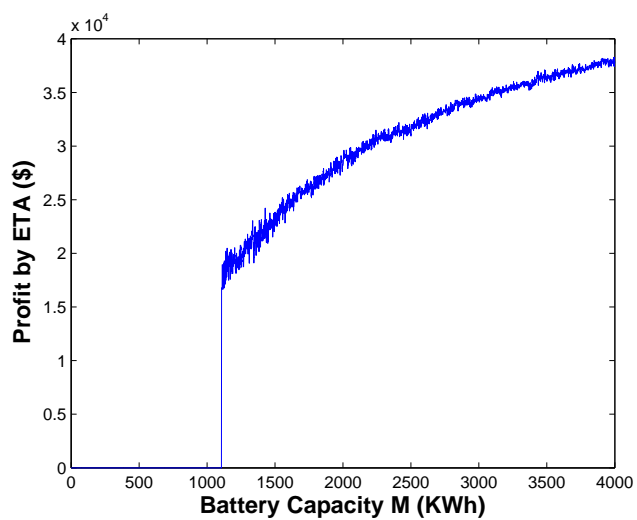

Fig. 11. Profit achieved by ETA in a community outperforms the heuristic scheme. It can be observed that the energy harvesting devices can save about $\$ 80$ in one year if there is no smart control. Nevertheless, with the help of ETA, it can further save $\$ 190$, which implies a total profit of $\$ 270$ per year.

\section{Discussion: Potential Applicability}

From an engineering perspective, our main contribution is to devise an energy trading scheme that can make profit for an end-user equipped with a battery. Yet it should be noted that $\$ 270$ per year is achieved under a large battery capacity and there is usually a wear-and-tear cost associated with the battery. More specifically, a battery only has a limited life. Thus, in this section, we will discuss the engineering potential of our scheme.

From Theorem 4.3, we note that the battery level is always less than $\theta+b_{\max }+r_{\max }=2 b_{\max }+r_{\max }+V P_{\max }$, which is because $\theta=b_{\max }+V P_{\max }$. Thus, for a given battery with capacity $M$, we can obtain $V=\frac{M-2 b_{\max }-r_{\max }}{P_{\max }}$. Note that in practice, $M$ is usually greater than $3 b_{\max }^{\max }$, which is the maximum possible amount of energy harvested in three hours (slots). Recall that we have $b_{\max } \geq r_{\max }$, so we obtain $M-2 b_{\max }-r_{\max } \geq 0$, which leads to a positive value of $V$. Therefore, we can implement our scheme ETA for a given battery size $M$.

First, let us focus on a single home. Fig. 10 illustrates the profit achieved by different values of battery capacity. The profit for a small battery size is equal to zero because the battery size is less than $3 b_{\max }$ and our scheme cannot be implemented. We observe that the largest $\frac{\text { Profit }}{\text { Battery capacity }}$ is $17 \$ / \mathrm{KWh}$, which is achieved when the battery capacity $M=5 \mathrm{KWh}$ and the corresponding profit is $\$ 85$ per year.

The cost of a lead-acid 5KWh battery is roughly $\$ 400-\$ 500$, and it has a lifetime of at least 5 years. The total profit achieved by ETA within five years is $\$ 425$, which is around the same as the battery cost. However, the average electricity price of the traces used here is only $0.05 \$ / \mathrm{KWh}$, which is less than the current average electricity price $0.12 \$ / \mathrm{KWh}$ in US. With a higher electricity price, we can expect a higher profit than $\$ 85$ per year. Furthermore, improvement in battery technology is in prospect, which may lead to lower costs and longer lifetime.

Next, we consider a community or an organization with a small solar farm, which has batteries with larger capacity and higher energy harvesting rate. In our simulation, we scale both energy harvesting rate and energy demand by 300 times. From the data shown in Fig. 11, we also observe that the largest $\frac{\text { Profit }}{\text { Battery capacity }}$ is $18 \$ / \mathrm{KWh}$, which is achieved when the battery capacity $M=1148 \mathrm{KWh}$ and the corresponding profit is $\$ 20485$ per year. Note that the largest value of $\frac{\text { Profit }}{\text { Battery capacity }}$ is greater than the previous single home case. The reason is that as we scale energy harvesting rate and energy demand, the parameter $V$ also scales, which leads to a better performance. Thus, we claim that compared to a single home, ETA has a greater potential of applicability for a community in the smart grid.

\section{CONCLUSION}

In this paper, we investigate the profit maximization problem for an end-user, which is equipped with an energy storage battery and an energy harvesting device. The price of electricity is time-varying, which represents an opportunity to make profit via buying the energy when the price is low and selling the energy when the price is high. Our proposed algorithm, ETA, achieves asymptotically optimal performance compared to a heuristic greedy scheme. Furthermore, it does not require any future information of the electricity prices, the energy demands and the renewable energy arrival process. We validate our results through rigorous trace driven simulations. Based on the simulation observations, we discuss the potential applicability of our scheme in both a typical home and a community setting by taking into account the wear-and-tear cost of the battery.

\section{REFERENCES}

[1] H. Farhangi, "The path of the smart grid," IEEE Power Energy Magazine, vol. 8, pp. 18-28, 2010.

[2] M. Liserre, T. Sauter, and J. Hung, "Future Energy Systems: Integrating Renewable Energy Sources into the Smart Power Grid Through Industrial Electronics," IEEE Industrial Electronics Magazine, vol. 4, pp. 18$37,2010$. 
[3] "California iso open access same-time information system (oasis) hourly average energy prices." http://oasisis.caiso.com.

[4] S. Chen, P. Sinha, and N. Shroff, "Scheduling heterogeneous delay tolerant tasks in smart grid with renewable energy," in CDC, Proceedings IEEE, 2012. To appear.

[5] I. Koutsopoulos and L. Tassiulas, "Control and optimization meet the smart power grid - scheduling of power demands for optimal energy management," Computing Research Repository, vol. abs/1008.3, 2010.

[6] M. J. Neely, A. S. Tehrani, and A. G. Dimakis, "Efficient algorithms for renewable energy allocation to delay tolerant consumers," in IEEE International Conference on Smart Grid Communications, 2010.

[7] A. Papavasiliou and S. S. Oren, "Supplying renewable energy to deferrable loads: Algorithms and economic analysis," in Power Engineering Society, IEEE General Meeting, pp. 1-8, 2010.

[8] A.-H. Mohsenian-Rad, V. W. S. Wong, J. Jatskevich, and R. Schober, "Optimal and autonomous incentive-based energy consumption scheduling algorithm for smart grid," in Innovative Smart Grid Technologies, 2010.

[9] P. Vytelingum, T. D. Voice, S. D. Ramchurn, A. Rogers, and N. R. Jennings, "Agent-based micro-storage management for the smart grid," in The Ninth International Conference on Autonomous Agents and Multiagent Systems (AAMAS 2010) - Won the Best Paper Award, pp. 3946, 2010. Winner of the Best Paper Award Event Dates: May 10-14, 2010.

[10] S. D. Ramchurn, P. Vytelingum, A. Rogers, and N. R. Jennings, "Agentbased homeostatic control for green energy in the smart grid," $A C M$ Trans. Intell. Syst. Technol., vol. 2, pp. 35:1-35:28, July 2011.

[11] A. Lam, L. Huang, A. Silva, and W. Saad, "A multi-layer market for vehicle-to-grid energy trading in the smart grid," in Computer Cотmunications Workshops (INFOCOM WKSHPS), 2012 IEEE Conference on, pp. $85-90$, march 2012.

[12] M. J. Neely, "Stock market trading via stochastic network optimization," in Conference on Decision and Control, pp. 2777-2784, 2010.

[13] M. J. Neely, Stochastic Network Optimization with Application to Communication and Queueing Systems. Morgan and Claypool Publishers, 2010.

[14] S. Chen, P. Sinha, and N. B. Shroff, "Energy Trading in the Smart Grid: From End-user's Perspective." http://www.ece.osu.edu/ chens/ chen13tech.pdf, 2012.

[15] "National renewable energy laboratory." http://www.nrel.gov.

[16] "Daily demand curve." http://www.charvolant.org/.

\section{APPENDIX A}

\section{PROOF OF THEOREM 4.3}

Proof: Part 1): We use induction to prove the result.

i). We have $B(0)=\theta<\theta+b_{\max }+r_{\max }$.

ii). Assume that $B(t-1) \leq \theta+b_{\max }+r_{\max }$ holds.

iii). For time slot $t$, we consider two subcases.

First, if $B(t-1) \leq \theta$, we can see that the maximum increase of the battery level during one time slot is $r_{\max }+b_{\max }$, which happens if $r(t)=r_{\max }$ and $b(t)=-b_{\max }$. Thus, we have $B(t) \leq \theta+b_{\max }+r_{\max }$.

Second, if $B(t-1)>\theta$, from Eqn. (9), we can see that $b(t)=b_{\text {max }}$ since $V \beta P(t)+B(t)-\theta \geq 0$. That is, as long as the battery level is greater than $\theta$, it discharges at rate $b_{\max }$. Note that $r(t) \leq r_{\max }$ and since we have that $r_{\max } \leq b_{\max }$, we obtain $r(t) \leq b_{\max }$. Hence, it follows that $B(t)=B(t-$ $1)+r(t)-b_{\max } \leq B(t-1) \leq \theta+r_{\max }+b_{\max }$. Therefore, we conclude that $B(t) \leq \theta+r_{\max }+b_{\max }$ holds for any $t$, which means that under ETA, the battery level is always bounded. Thus, the required battery size is finite.

Part 2): Recall that ETA minimizes the RHS of Eqn. (12). We compare the stationary and randomized policy in Lemma 3.1 and our dynamic energy trading scheme ETA. In particular, we have that

$$
\begin{aligned}
& D+(V P(t)+B(t)-\theta) \mathbb{E}\left[\left(d(t)-b^{*}(t)\right)^{+} \mid B(t)\right] \\
& -(V \beta P(t)+B(t)-\theta) \mathbb{E}\left[\left(d(t)-b^{*}(t)\right)^{-} \mid B(t)\right] \\
& -(B(t)-\theta)(\bar{d}-\bar{r}) \\
& \leq D+(V P(t)+B(t)-\theta) \mathbb{E}\left[(d(t)-\tilde{b}(t))^{+} \mid B(t)\right] \\
& -(V \beta P(t)+B(t)-\theta) \mathbb{E}\left[(d(t)-\tilde{b}(t))^{-} \mid B(t)\right] \\
& -(B(t)-\theta)(\bar{d}-\bar{r}) \\
& =D+(B(t)-\theta) \mathbb{E}[(d(t)-\tilde{b}(t)) \mid B(t)] \\
& -V \mathbb{E}\left[\beta P(t)(d(t)-\tilde{b}(t))^{-}-P(t)(d(t)-\tilde{b}(t))^{+} \mid B(t)\right] \\
& -(B(t)-\theta)(\bar{d}-\bar{r}),
\end{aligned}
$$

where we have used the fact $f=f^{+}-f^{-}, \forall f$. Recalling that $d(t)$ and $\tilde{b}(t)$ are independent of $B(t)$ and $\mathbb{E}[\tilde{b}(t)]=\bar{r}$ in Lemma 3.1, we have $\mathbb{E}[(d(t)-\tilde{b}(t)) \mid B(t)]=\bar{d}-\bar{r}$. Thus, the RHS of Eqn. (14) can be rewritten as

$$
\begin{aligned}
& D+(B(t)-\theta) \mathbb{E}[(d(t)-\tilde{b}(t)) \mid B(t)] \\
& -V \mathbb{E}\left[\beta P(t)(d(t)-\tilde{b}(t))^{-}-P(t)(d(t)-\tilde{b}(t))^{+} \mid B(t)\right] \\
& -(B(t)-\theta)(\bar{d}-\bar{r}) \\
& =D-V \mathbb{E}\left[\beta P(t)(d(t)-\tilde{b}(t))^{-}-P(t)(d(t)-\tilde{b}(t))^{+}\right] .
\end{aligned}
$$

Thus, combining Eqns. (12), (14), (15), we have

$$
\begin{aligned}
& \mathbb{E}[(L(t+1)-L(t) \mid B(t)]- \\
& V \mathbb{E}\left[\beta P(t)\left(d(t)-b^{*}(t)\right)^{-}-P(t)\left(d(t)-b^{*}(t)\right)^{+} \mid B(t)\right] \\
& \leq D-V \mathbb{E}\left[\beta P(t)(d(t)-\tilde{b}(t))^{-}-P(t)(d(t)-\tilde{b}(t))^{+}\right] .
\end{aligned}
$$

By taking expectations with respect to $B(t)$ on both sides of Eqn. (16) and using the fact $\mathbb{E}[\mathbb{E}[X \mid Y]]=\mathbb{E}[X], \forall X, Y$, we take the summation from $t=0$ to $T$ and obtain that

$$
\begin{aligned}
& \mathbb{E}[(L(T+1)-L(0)]- \\
& \sum_{i=1}^{T} V \mathbb{E}\left[\beta P(t)\left(d(t)-b^{*}(t)\right)^{-}-P(t)\left(d(t)-b^{*}(t)\right)^{+}\right] \\
& \leq T D-V \sum_{t=1}^{T} \mathbb{E}\left[\beta P(t)(d(t)-\tilde{b}(t))^{-}-P(t)(d(t)-\tilde{b}(t))^{+}\right] .
\end{aligned}
$$

If we set $B(0)=\theta$, we have $L(0)=0$. Rearranging Eqn. (17) and dividing by $V T$ on both sides, we have

$$
\begin{aligned}
& \frac{1}{T} \sum_{t=1}^{T} \mathbb{E}\left[\beta P(t)\left(d(t)-b^{*}(t)\right)^{-}-P(t)\left(d(t)-b^{*}(t)\right)^{+}\right] \\
& \geq \frac{1}{T} \sum_{t=1}^{T} \mathbb{E}\left[\beta P(t)(d(t)-\tilde{b}(t))^{-}-P(t)(d(t)-\tilde{b}(t))^{+}\right]-\frac{D}{V}
\end{aligned}
$$


Taking the limsup as $T \rightarrow \infty$ and using the result in Lemma 3.1, we obtain

$$
\begin{aligned}
& \limsup _{T \rightarrow \infty} \frac{1}{T} \sum_{t=1}^{T} \mathbb{E}\left[\beta P(t)(d(t)-b(t))^{-}-P(t)(d(t)-b(t))^{+}\right] \\
& \geq \tilde{c}-\frac{D}{V} \geq c^{\text {opt }}-\frac{D}{V}
\end{aligned}
$$

where we use $\tilde{c} \geq c^{o p t}$ for the last inequality. 